\title{
Memristor MOS Content Addressable Memory (MCAM): Hybrid Architecture for Future High Performance Search Engines
}

\author{
Kamran Eshraghian, Kyoung-Rok Cho, Member, IEEE, Omid Kavehei, Student Member, IEEE, Soon-Ku Kang, \\ Derek Abbott, Fellow, IEEE, and Sung-Mo Steve Kang, Fellow, IEEE
}

\begin{abstract}
Large-capacity content addressable memory (CAM) is a key element in a wide variety of applications. The inevitable complexities of scaling MOS transistors introduce a major challenge in the realization of such systems. Convergence of disparate technologies, which are compatible with CMOS processing, may allow extension of Moore's Law for a few more years. This paper provides a new approach towards the design and modeling of Memory resistor (Memristor)-based CAM (MCAM) using a combination of memristor MOS devices to form the core of a memory/compare logic cell that forms the building block of the CAM architecture. The non-volatile characteristic and the nanoscale geometry together with compatibility of the memristor with CMOS processing technology increases the packing density, provides for new approaches towards power management through disabling CAM blocks without loss of stored data, reduces power dissipation, and has scope for speed improvement as the technology matures.
\end{abstract}

Index Terms-Content addressable memory (CAM), memory, memory resistor-based CAM (MCAM), memory resistor (memristor)-MOS hybrid architecture, modeling.

\section{INTRODUCTION}

$\mathbf{T}$ HE QUEST for a new hardware paradigm that will attain processing speeds in the order of an exaflop $\left(10^{18}\right.$ floating point operations per second) and further into the zetaflop regime $\left(10^{21}\right.$ flops) is a major challenge for both circuit designers and system architects. The evolutionary progress of networks such as the Internet also brings about the need for realization of new components and related circuits that are compatible with CMOS process technology as CMOS scaling begins to slow down [1].

Manuscript received December 31, 2009; revised March 15, 2010; accepted April 20, 2010. Date of publication May 24, 2010; date of current version July 27, 2011. This work was supported by Grant R33-2008-000-1040-0 from the World Class University (WCU) Project of MEST and KOSEF through Chungbuk National University (CBNU).

K. Eshraghian, K. R. Cho, and S. K. Kang are with the College of Electrical and Information Engineering, WCU Program, Chungbuk National University, Cheongju 361-763, South Korea (e-mail: k.eshraghian@innovationlabs. com.au; krcho@cbnu.ac.kr; skkang@hbt.cbnu.ac.kr).

O. Kavehei is with the College of Electrical and Information Engineering, WCU Program, Chungbuk National University, Cheongju 361-763, South Korea, and also with the School of Electrical and Electronic Engineering, University of Adelaide, Adelaide SA 5005, Australia (e-mail: omid@ hbt.cbnu.ac.kr; omid@eleceng.adelaide.edu.au).

D. Abbott is with the School of Electrical and Electronic Engineering, University of Adelaide, Adelaide SA 5005, Australia (e-mail: dabbott@eleceng.adelaide.edu.au).

S. M. Kang is with the School of Engineering, University of California, Merced, CA 95343 USA (e-mail: smk123@ucmerced.edu).

Digital Object Identifier 10.1109/TVLSI.2010.2049867
As Moore's Law becomes more difficult to fulfill, integration of significantly different technologies such as spintronics [1], carbon nano tube field effect transistors (CNFET) [2], optical nanocircuits based on metamaterials [3], and more recently the memristor [4], are gaining more focus thus creating new possibilities towards realization of innovative circuits and systems within the system on system $(\mathrm{SoS})$ domain.

In this paper we explore conceptualization, design, and modeling of the memory/compare cell as part of a memristor-based content addressable memory (MCAM) architecture using a combination of memristor and n-type MOS devices. A typical content addressable memory (CAM) cell forms a SRAM cell that has two n-type and two p-type MOS transistors, which requires both $V_{\mathrm{DD}}$ and GND connections as well as well-plugs within each cell. Construction of a SRAM cell that exploits memristor technology, which has a non-volatile memory (NVM) behavior and can be fabricated as an extension to a CMOS process technology with nanoscale geometry, addresses the main thread of current CAM research towards reduction of power consumption.

The design of the CAM cell is based on the fourth passive circuit element, the memristor (M) predicted by Chua in 1971 [5] and generalized by Kang [6], [7]. Chua postulated that a new circuit element defined by the single-valued relationship $d \phi=M d q$ must exist, whereby current moving through the memristor is proportional to the flux of the magnetic field that flows through the material. In another words, the magnetic flux between the terminals is a function of the amount of charge, $q$, that has passed through the device. This follows from Lenz's law whereby the single-valued relationship $d \phi=M d q$ has the equivalence $v=M(q) i$, where $v$ and $i$ are memristor voltage and current, respectively.

The memristor behaves as a switch, much like a transistor. However, unlike the transistor, it is a two-terminal rather than a three-terminal device and does not require power to retain either of its two states. Note that a memristor changes its resistance between two values and this is achieved via the movement of mobile ionic charge within an oxide layer, furthermore, these resistive states are non-volatile. This behavior is an important property that influences the architecture of CAM systems, where the power supply of CAM blocks can be disabled without loss of stored data. Therefore, memristor-based CAM cells have the potential for significant saving in power dissipation.

This paper has the following structure. Section II is an introductory section and reviews the properties of the memristor and then explores various options available in the modeling of this 


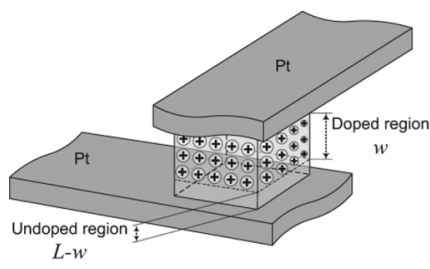

(a)

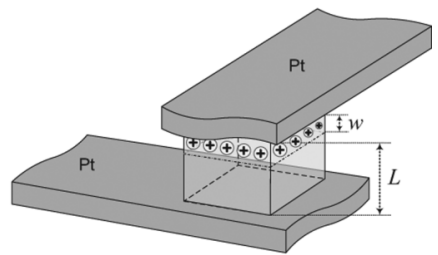

(b)
Fig. 1. Memristor switching behavior. (a) "ON" state, low resistance, (b) "OFF" state, high resistance. The key feature of memristor is it can remember the resistance once the voltage is disconnected. In (a) "doped" and "undoped" regions are related to $R_{\mathrm{ON}}$ and $R_{\mathrm{OFF}}$, respectively. The dopant consists of mobile charges. In (b), $L$ and $w$ are the thin-film thickness and doped region thickness, respectively.

device. In Section III, circuit options for realization of MCAM is investigated whereby the two disparate technologies converge to create a new CMOS-based design platform. Section IV provides simulation results of a basic MCAM cell to be implemented as part of a future search engine. The details of our proposed layout and preliminary CMOS overlay fabrication approach are also presented in Section V. The concluding comments are provided in Section VI.

\section{Characterization AND MODEling BeHAVIOR OF MEMRISTOR}

Strukov et al. [4] presented a physical model whereby the memristor is characterized by an equivalent time-dependent resistor whose value at a time $t$ is linearly proportional to the quantity of charge $q$ that has passed through it. They realized a proof-of-concept memristor, which consists of a thin nano layer $(2 \mathrm{~nm})$ of $\mathrm{TiO}_{2}$ and a second oxygen deficient nano layer of $\mathrm{TiO}_{2-x}(8 \mathrm{~nm})$ sandwiched between two Pt nanowires $(\sim$ $50 \mathrm{~nm})$, shown in Fig. 1 [4]. Oxygen $\left(\mathrm{O}^{2-}\right)$ vacancies are +2 mobile carriers and are positively charged. A change in distribution of $\mathrm{O}^{2-}$ within the $\mathrm{TiO}_{2}$ nano layer changes the resistance. By applying a positive voltage, to the top platinum nanowire, oxygen vacancies drift from the $\mathrm{TiO}_{2-x}$ layer to the $\mathrm{TiO}_{2}$ undoped layer, thus changing the boundary between the $\mathrm{TiO}_{2-x}$ and $\mathrm{TiO}_{2}$ layers. As a consequence, the overall resistance of the layer is reduced corresponding to an "ON" state. When enough charge passes through the memristor that ions can no longer move, the device enters a hysteresis region and keeps $q$ at an upper bound with fixed memristance, $M$ (memristor resistance). By reversing the process, the oxygen defects diffuse back into the $\mathrm{TiO}_{2-x}$ nano layer. The resistance returns to its original state, which corresponds to an "OFF" state. The significant aspect to be noted here is that only ionic charges, namely oxygen vacancies $\left(\mathrm{O}^{2-}\right)$ through the cell, change memristance. The resistance change is non-volatile hence the cell acts as a memory element that remembers past history of ionic charge flow through the cell.

\section{A. Simplified Memristor Model}

The memristor can be modeled in terms of two resistors in series, namely the doped region and undoped region each having vertical width of $w$ and $L-w$, respectively, as shown in Fig. 1, where $L$ is the $\mathrm{TiO}_{2}$ film thickness [4]. The voltage-current relationship defined as $M(q)$, can be modeled as [5]

$$
v(t)=\left(R_{\mathrm{ON}} \frac{w(t)}{L}+R_{\mathrm{OFF}}\left(1-\frac{w(t)}{L}\right)\right) i(t)
$$

where $R_{\mathrm{ON}}$ is the resistance for completely doped memristor, while $R_{\mathrm{OFF}}$ is the resistance for the undoped region. The width of the doped region $w(t)$ is given by

$$
\frac{d w(t)}{d t}=\mu_{v} \frac{R_{\mathrm{ON}}}{L} i(t)
$$

where $\mu_{v}$ represents the average dopant mobility $\sim 10^{-10} \mathrm{~cm}^{2} / \mathrm{s} / \mathrm{V}$. Taking a normalized variable, $x(t)=w(t) / L$, instead of $w(t)$ assists in tracking memristance, $M(q)=d \phi / d q$, or memductance, $W(\phi)=d q / d \phi$. The new normalized relation is

$$
\frac{d x(t)}{d t}=\mu_{v} \frac{R_{\mathrm{ON}}}{L^{2}} i(t)
$$

where $L^{2} / \mu_{v}$ has the dimensions of magnetic flux $(\phi)$. Following the calculation steps from Kavehei et al. [8], a simple memristance model can be defined as

$$
M(t)=R_{\mathrm{OFF}}\left(\sqrt{1-\frac{2 c(t)}{r}}\right)
$$

where $c(t)=\mu_{v} \phi(t) / L^{2}$, and $r$ is a ratio of $R_{\mathrm{OFF}} / R_{\mathrm{ON}}$ and $\sqrt{1-2 c(t) / r}$ is the resistance modulation index. Here, $x(t)$ can now be rewritten as

$$
x(t)=1-\left(\sqrt{1-\frac{2 \phi(t)}{r \beta}}\right)
$$

which highlights that the $r \beta$ term (where $\beta=L^{2} / \mu_{v}$ ) must be made sufficiently large to maintain $2 \phi(t) / r \beta$ between the range 0 and 1 . The simplified linear ionic drift model facilitates the understanding of the operational characteristics of the memristor. However, for a highly nonlinear [9] relationship between electric field and drift velocity that exists at the boundaries, the ratio cannot be maintained. Thus this function is unable to model large nonlinearities close to the boundaries of the memristor characteristics. At the boundaries, i.e., when $x$ approaches 0 or 1 , there is a nonlinearity associated with the memristor behavior that is discussed in Section II-B.

\section{B. Modelling the Nonlinear Behavior of Memristor}

The electrical behavior of the memristor as a switch/memory element is determined by the boundary between the two regions in response to an applied voltage. To model this nonlinearity, the memristor state (3) is augmented with a window function, $F(w, i)[4],[10]-[12]$, where $w$ and $i$ are the memristor's state variable and current, respectively.

Thus, (3) can be rewritten as

$$
\frac{d x(t)}{d t}=\frac{R_{\mathrm{ON}}}{\beta} i(t) F(x(t), p)
$$


TABLE I

Comparison Between Different Memristor Models. For A-II, B-I, AND B-II $x=w / L$

\begin{tabular}{|c|c|c|c|c|}
\hline Model & Ref & $\begin{array}{c}\text { Window Function } \\
F(\cdot)\end{array}$ & $\begin{array}{c}\text { Boundaries } \\
(x \rightarrow 0, x \rightarrow 1)\end{array}$ & Problem(s) \\
\hline$\overline{\mathrm{A}-\mathrm{I}}$ & [4] & $\bar{w} w(1-w) / L^{2}$ & $(0, \sim 0)$ & $\begin{array}{l}\text { Linear approximation, } x \in[0,1] \\
\text { Stuck at the terminal states } \\
F(w \rightarrow L) \neq 0\end{array}$ \\
\hline A-II & [12] & $x(1-x)$ & $(0,0)$ & $\begin{array}{l}\text { Linear approximation, } x \in[0,1] \\
\text { Stuck at the terminal states }\end{array}$ \\
\hline B-I & [13] & $1-(2 x-1)^{2 p}$ & $(0,0)$ & Stuck at the terminal states \\
\hline B-II* & [11] & $1-(x-\operatorname{sgn}(-i))^{2 p}$ & $(0,0)$ & Discontinuity at the boundaries \\
\hline
\end{tabular}

* This model is adopted for the simulations.

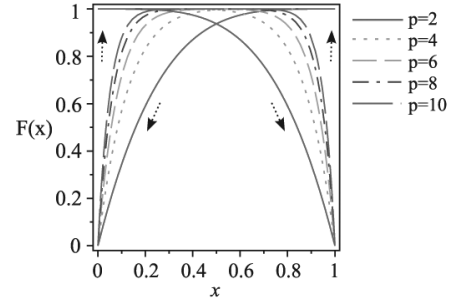

(a)

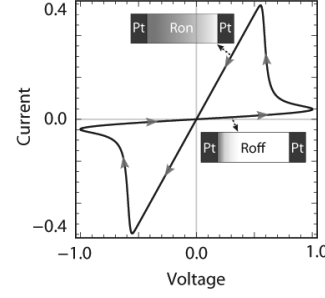

(b)
Fig. 2. Nonlinear behavior of the memristor. (a) Window function: $F(x)=$ $1-(x-\operatorname{sgn}(-i))^{2 p}$, where $\operatorname{sgn}(I)$ gives the sign of the input signal $I$. (b) The hysteresis characteristics using the nonlinear drift assumption. This hysteresis shows a highly nonlinear relationship between current and voltage at the boundaries.

where $p$ is its control parameter. The nonlinearity at the boundaries can now be controlled with parameter $p$. The influence of a window function described by (6) is illustrated in Fig. 2(a) for $2 \leq p \leq 10$.

Joglekar and Wolf [13] proposed a modified window function to approximately address linear ionic drift and the nonlinear behaviour at the boundaries when $0<x<1$. For the window function $F(x)=1-(2 x-1)^{2 p}, p$ is a positive integer and $x=w / L$. This model considers a simple boundary condition, $F(0)=F(1)=0$, when $p \geq 4$, the state variable equation is an approximation of the linear drift assumption, $F(0<x<1) \approx 1$. This model is denoted by B-I in Table I.

Based on this model, when a memristor is at the terminal states, no external stimulus can change its state. Biolek et al. [11] addressed this problem with a new window function, $F(x)=1-(x-\operatorname{sgn}(-i))^{2 p}$, where $i$ is the memristor current, $\operatorname{sgn}(i)=1$ when $i \geq 0$, and $\operatorname{sgn}(i)=0$ when $i<0$. When current is positive, the doped region length, $w$, is expanding. This model is denoted by B-II in Table I and is adopted for the simulations that follow.

The hysteresis characteristic using the nonlinear drift assumption is illustrated in Fig. 2(b). This hysteresis shows a highly nonlinear relationship between current and voltage at the boundaries as is derived using similar parameters reported by Strukov et al. [4]

To conclude this section Table I shows a brief comparison between different behavioral memristor models. It is also important to emphasis that the modeling approach in this paper is based on the behavioral characteristics of the solid-state thin film memristor device [4]. Shin et al. [14] recently proposed compact macromodels for the solid-state thin film memristor device. Even though the assumption is still based on the linear drift model, their approach provides a solution for bypassing current flow at the two boundary resistances.

\section{Emerging Memory Devices and Technologies}

Memory processing has been considered as the pace-setter for scaling a technology. A number of performance parameters including capacity (that relate to area utilization), cost, speed (both access time and bandwidth), retention time, and persistence, read/write endurance, active power dissipation, standby power, robustness such as reliability and temperature related issues characterize memories. Recent and emerging technologies such as phase-change random access memory (PCRAM), magnetic RAM (MRAM), ferroelectric RAM (FeRAM), resistive RAM (RRAM), and memristor, have shown promise and some are already being considered for implementation into emerging products. Table II summarizes a range of performance parameters and salient features of each of the technologies that characterize memories [15], [16]. A projected plan for 2020 for memories highlight a capacity greater than $1 \mathrm{~TB}$, read/write access times of less than $100 \mathrm{~ns}$ and endurance in the order of $10^{12}$ or more write cycles.

Flash memories suffer from both a slow write/erase times and low endurance cycles. FeRAMs and MRAMs are poorly scalable. MRAMs and PCRAMs require large programming currents during write cycle, hence an increase in dissipation per bit. Furthermore, voltage scaling becomes more difficult. Memristors, however, have demonstrated promising results in terms of the write operation voltage scaling [10], [17].

Memristor crossbar-based architecture is highly scalable [18] and shows promise for ultra-high density memories [19]. For example, a memristor with minimum feature sizes of 10 and 3 $\mathrm{nm}$ yield $250 \mathrm{~Gb} / \mathrm{cm}^{2}$ and $2.5 \mathrm{~Tb} / \mathrm{cm}^{2}$, respectively.

In spite of the high density, zero standby power dissipation, and long life time that have been pointed out for the emerging memory technologies, their long write latency has a large negative source of impact on memory bandwidth, power consumption, and the general performance of a memory system.

\section{Conventional CAM And the Proposed MCAM STRUCTURES}

A content addressable memory illustrated in Fig. 3 takes a search word and returns the matching memory location. Such an approach can be considered as a mapping of the large space 
TABLE II

Traditional AND EMERGing MEMORY TECHNOLOGIES

\begin{tabular}{|c|c|c|c|c|c|c|c|c|}
\hline & \multicolumn{4}{|c|}{ Traditional Technologies } & \multicolumn{4}{|c|}{ Emerging Technologies } \\
\hline & & & Imprc & d Flash & & & & \\
\hline & DRAM & SRAM & NOR & NAND & FeRAM & MRAM & PCRAM & Memristor \\
\hline Knowledge level & \multicolumn{2}{|c|}{ mature } & \multicolumn{2}{|c|}{ advanced } & \multicolumn{2}{|c|}{$\begin{array}{l}\text { product } \\
\end{array}$} & advanced & early stage \\
\hline Cell Elements & 1T1C & $6 \mathrm{~T}$ & \multicolumn{2}{|c|}{$1 \mathrm{~T}$} & 1T1C & 1T1R & 1T1R & $1 \mathrm{M}$ \\
\hline Half pitch $(F)(\mathrm{nm})$ & 50 & 65 & 90 & 90 & 180 & 130 & 65 & $3-10$ \\
\hline Smallest cell area $\left(F^{2}\right)$ & 6 & 140 & 10 & 5 & 22 & 45 & 16 & 4 \\
\hline Read time (ns) & $<1$ & $<0.3$ & $<10$ & $<50$ & $<45$ & $<20$ & $<60$ & $<50$ \\
\hline Write/Erase time (ns) & $<0.5$ & $<0.3$ & $10^{5}$ & $10^{6}$ & 10 & 20 & 60 & $<250$ \\
\hline Retention time (years) & seconds & N/A & $>10$ & $>10$ & $>10$ & $>10$ & $>10$ & $>10$ \\
\hline Write op. voltage (V) & 2.5 & 1 & 12 & 15 & $0.9-3.3$ & 1.5 & 3 & $<3$ \\
\hline Read op. voltage (V) & 1.8 & 1 & 2 & 2 & $0.9-3.3$ & 1.5 & 3 & $<3$ \\
\hline Write endurance & $10^{16}$ & $10^{16}$ & $10^{5}$ & $10^{5}$ & $10^{14}$ & $10^{16}$ & $10^{9}$ & $10^{15}$ \\
\hline Write energy (fJ/bit) & 5 & 0.7 & 10 & 10 & 30 & $1.5 \times 10^{5}$ & $6 \times 10^{3}$ & $<50$ \\
\hline Density $\left(\mathrm{Gbit} / \mathrm{cm}^{2}\right)$ & 6.67 & 0.17 & 1.23 & 2.47 & 0.14 & 0.13 & 1.48 & 250 \\
\hline Voltage scaling & & & y scal & & & no & poor & promising \\
\hline Highly scalable & maj & technol & cal ba & & & & promising & promising \\
\hline
\end{tabular}

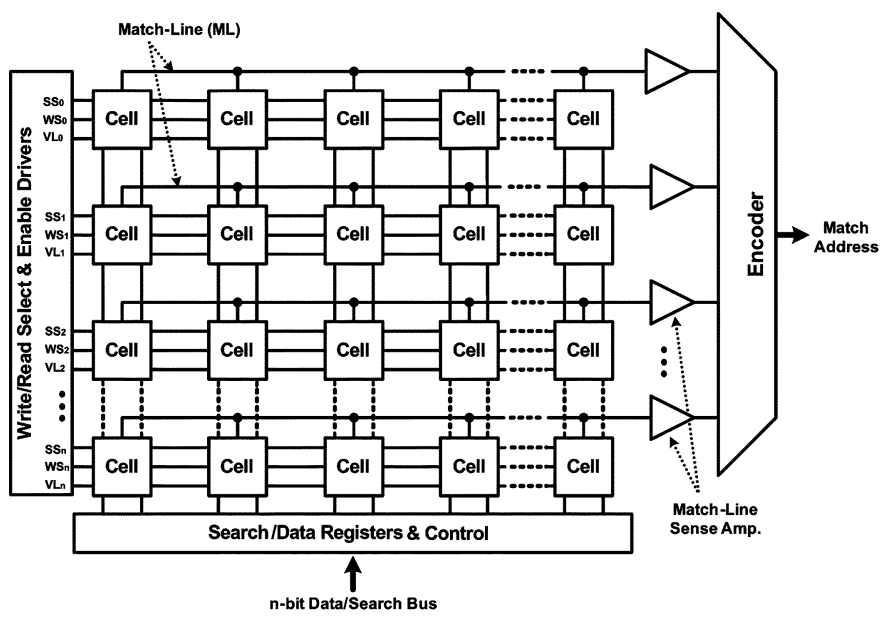

Fig. 3. Generic content addressable memory architecture for $n \times n$ NAND-type CAM cells. In this structure each data (D) and search (S) bits share one common bus line $(\mathrm{D} / \mathrm{S})$ to reduce the interconnection complexity. The architecture is based on the MCAM cell of Fig. 6(d) and the match lines (MLs) composed of nMOS pass transistors.

of input search word to that of the smaller space of output match location in a single clock cycle [20]. There are numerous applications including translation lookaside buffers (TLB), image coding [21], classifiers to forward Internet protocol (IP) packets in network routers [22], etc. Inclusion of memristors in the architecture ensures that data is retained if the power source is removed enabling new possibilities in system design including the all important issue of power management.

\section{A. Conventional CAM}

To better appreciate some of the benefits of our proposed structure we provide a brief overview of the conventional CAM cell using static random access memory (SRAM) as shown in Fig. 4(a). The two inverters that form the latch use four transistors including two p-type transistors that normally require more silicon area. Problems such as relatively high leakage current particularly for nanoscaled CMOS technology [23] and the need for inclusion of both $V_{\mathrm{DD}}$ and ground lines in each cell bring further challenges for CAM designers in order to increase the packing density and still maintain sensible power dissipation. Thus, to satisfy the combination of ultra dense designs, low-

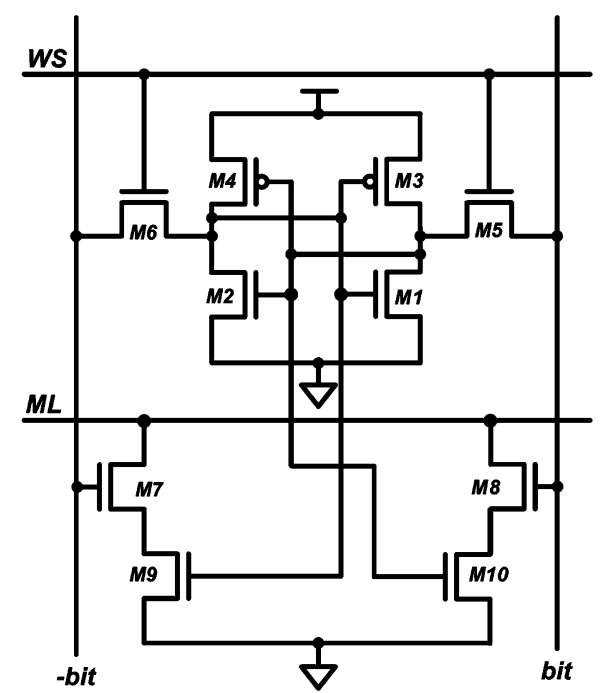

(a)

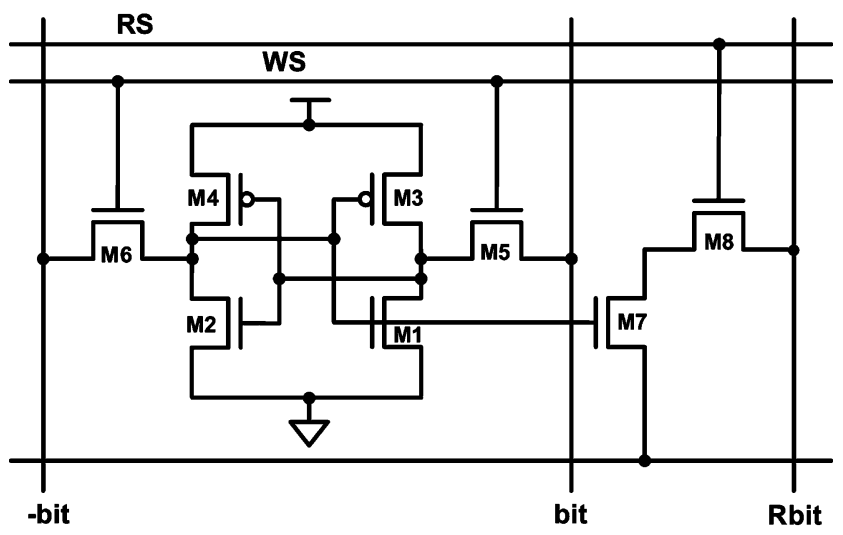

(b)

Fig. 4. Conventional CAM cell structure and the design of a SRAM cell for ultra low-power applications. In (a) a conventional 10-T NOR-type CAM circuit is demonstrated. Usually, conventional NOR- or NAND-type CAM cells have more than 9 transistors [26]. In (a) and (b), RS, Rbit, WS, ML, bit, and -bit lines are read select, read bit-line, word select, match line, data, and complementary data signals. (a) Conventional 10-T NOR-type CAM Cell. (b) 8-T subthreshold SRAM Cell [23].

power (low-leakage), and high-performance, the SRAM cell is the focus of architectural design considerations. 
For instance, one of the known problems of the conventional 6-T SRAM for ultra low-power applications is its static noise margin (SNM) [23]. Fundamentally, the main technique used to design an ultra low-power memory is voltage scaling that brings CMOS operation down to the subthreshold regime. Verma and Chandrakasan [23] demonstrated that at very low supply voltages the static noise margin for SRAM will disappear due to process variation. To address the low SNM for subthreshold supply voltage Verma and Chandrakasan [23] proposed 8-T SRAM cell shown in Fig. 4(b). This means, there is a need for significant increase in silicon area to have reduced failure when the supply voltage has been scaled down.

Failure is a major issue in designing ultra dense (high capacity) memories. Therefore, a range of fault tolerance techniques are usually applied [24]. As long as the defect or failure results from the SRAM structure, a traditional approach such as replication of memory cells can be implemented. Obviously it causes a large overhead in silicon area which, exacerbates the issue of power consumption.

Some of the specific CAM cells, for example, ternary content addressable memory (TCAM) normally used for the design of high-speed lookup-intensive applications in network routers, such as packet forwarding and classification two SRAM cells, are required. Thus, the dissipation brought about as the result of leakage becomes a major design challenge in TCAMs [25]. It should be noted that the focus in this paper is to address the design of the store/compare core cell only, leaving out details of CAM's peripherals such as read/write drivers, encoder, matchline sensing selective precharge, pipelining, matchline segmentation, current saving technique, etc., that characterize a CAM architecture [26].

\section{B. Generic Memristor-nMOS Circuit}

Fig. 5 shows the basic structure for a memristor-nMOS storage cell. For writing a logic " 1 ," the memristor receives a positive bias to maintain an "ON" state. This corresponds to the memristor being programmed as a logic " 1 ." To program a " 0 " a reverse bias is applied to the memristor, which makes the memristor resistance high. This corresponds to logic "0" being programmed.

\section{MCAM Cell}

In this subsection, variations of MCAM cells as well as a brief architectural perspective are introduced. The details of $\mathrm{read} / \mathrm{write}$ operations and their timing issues are also discussed in Section IV. A CAM cell serves two basic functions: "bit storage" and "bit comparison." There are a variety of approaches in the design of basic cell such as NOR-based match line, NAND based match line, etc. This part of the paper reviews the properties of conventional SRAM-based CAM and provides a possible approach for the design of content addressable memory based on the memristor.

1) MCAM Cell Properties: Fig. 6 illustrates several variations of the MCAM core whereby bit-storage is implemented by memristors ME1 and ME2. Bit comparison is performed by either NOR or alternatively NAND-based logic as part of the match-line $\mathrm{ML}_{i}$ circuitry. The matching operation is equivalent

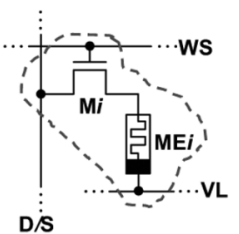

(a)

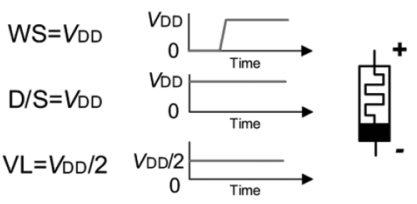

(c)

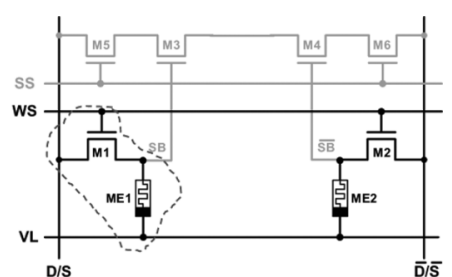

(b)

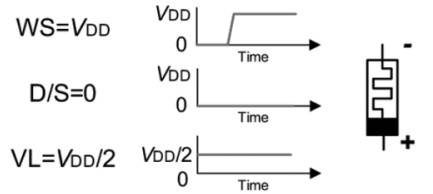

(d)
Fig. 5. Basic memristor-nMOS storage cell and the timing diagram. (a) shows write mode part of the $i$ th cell in a row. (b) Basic cell circuit without the matchline transistor. (c) "Low" resistance, $R_{\mathrm{ON}}$, programing. Equivalent to logic "1." (d) "High" resistance, $R_{\mathrm{OFF}}$, programing. Equivalent to logic "0." (a) Structure of write mode. (b) Basic cell. (c) Program "Low" resistance "1." (d) Program "High" resistance " $0 . "$

to logical XORing of the search bit (SB) and stored bit (D). The match-line transistors (ML) in the NOR-type cells can be considered as part of a pull-down path of a precharged NOR gate connected at the end of each individual $\mathrm{ML}_{i}$ row. The NAND-type CAM functions in a similar manner forming the pull-down of a precharged NAND gate. Although each of the selected cells in Fig. 6 have their relative merits, the approach in Fig. 6(c) where Data bits and Search bits share a common bus is selected for detailed analysis. The structure of the 7-T NAND-type, shown in Fig. 6(d), and the NOR-type are identical except for the position of the ML transistor. In the NOR-type, ML makes a connection between shared ML and ground while in the NAND-type, the ML transistors act as a series of switches between the $\mathrm{ML}_{i}$ and $\mathrm{ML}_{i+1}$.

\section{Simulation Results ANalysis AND Comparison}

Generally, there are the "write" and "read" operations that require consideration. In this section the "write" and "read" operations of the basic MCAM cell for 7-T NOR-type are reported. Simulations of the circuits are based on the following parameters [27]: $R_{\mathrm{ON}}=100 \Omega, R_{\mathrm{OFF}}=100 \mathrm{k} \Omega, p=4$, $L=3 \mathrm{~nm}$, and $\mu_{v}=3 \times 10^{-8} \mathrm{~m}^{2} / \mathrm{s} / \mathrm{V}$. Both the conventional CAM and MCAM circuits have been implemented using Dongbu HiTech $0.18-\mu \mathrm{m}$ technology where $1.8 \mathrm{~V}$ is the nominal operating voltage for the CAM. The MCAM cell is implemented using nMOS devices and memristors without the need for $V_{\mathrm{DD}}$ voltage source. Using the above memristor parameters, together with the behavioral model B-II of Table I, satisfactory operation of the MCAM cell is achieved at 3.0 Volts. We have referred to this voltage as the nominal voltage for the MCAM cell. Furthermore, the initial state of the memristors ("ON," "OFF," or in between) is determined by initial resistance, $R_{\mathrm{INIT}}$.

\section{A. Write Operation}

At the write phase, the memristor ME1 is programmed based on the data bit on the $\mathrm{D}$ line. The complementary data is also stored in ME2. During the write operation, the select line is zero 


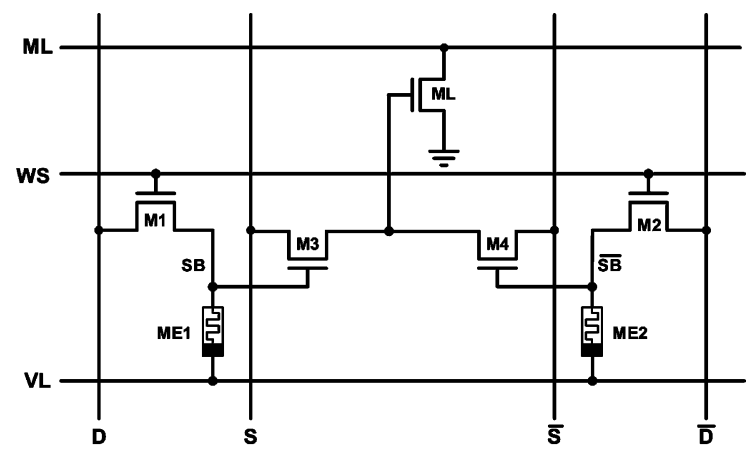

(a)

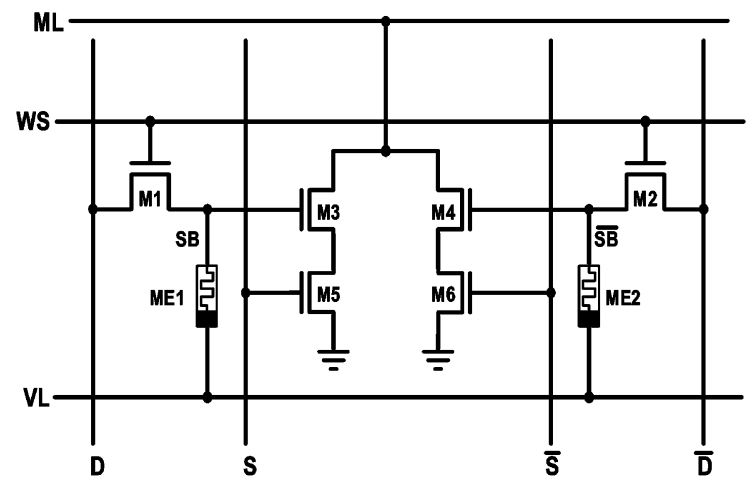

(b)

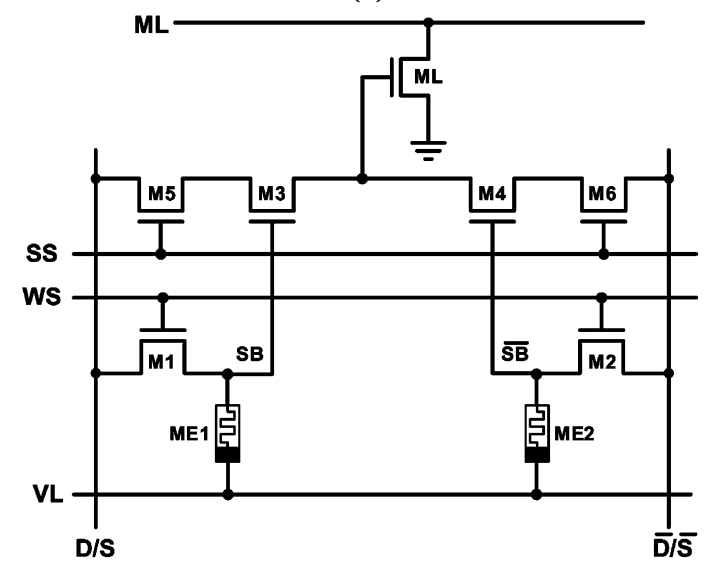

(c)

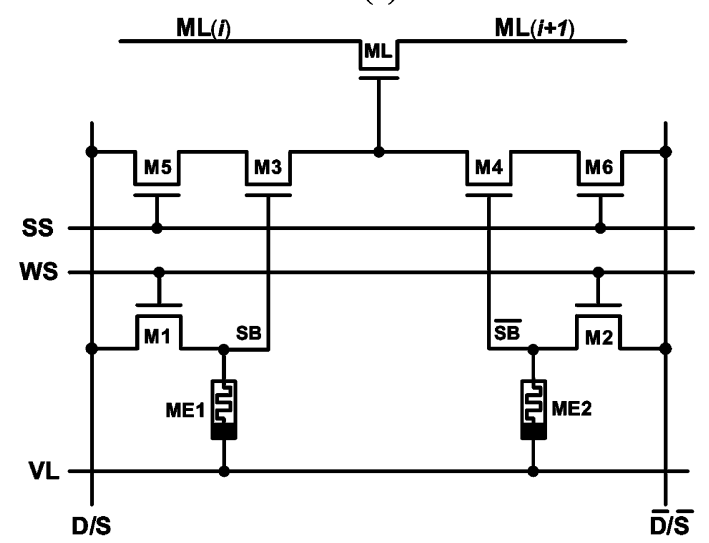

(d)

Fig. 6. Cell configurations of possible MCAM structures. (a) 5-T NOR-type. (b) 6-T NOR-type. (c) 7-T NOR-type. (d) 7-T NAND-type.

and an appropriate write voltage is applied on VL. The magnitude of this voltage is half of supply voltage, that corresponds

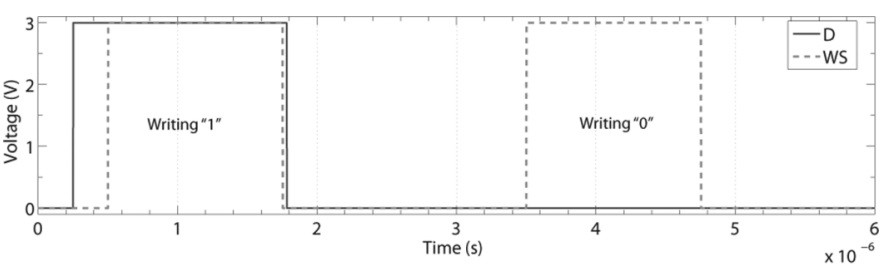

(a)

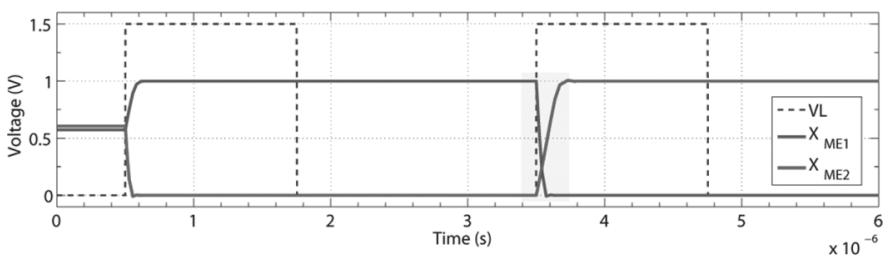

(b)

Fig. 7. Write operation timing diagram. The highlighted area in (b) shows the minimum time for writing, which is the maximum for both memristors, around $220 \mathrm{~ns}$. In (b) $x_{\mathrm{ME} 1}$ and $x_{\mathrm{ME} 2}$ are dimensionless parameters and both are varying between 0 and 1 . The rational for showing $\mathrm{VL}$ and $x_{\mathrm{ME} 1}$ and $x_{\mathrm{ME} 2}$ together is that $\mathrm{VL}$ acts as a trigger for the state variables. $\mathrm{VL}_{\text {active }}=1.5 \mathrm{~V}\left(V_{\mathrm{DD}} / 2\right)$ for write operation. (a) Data (D) and Word Select (WS) signals. WS pulse width is $1.2 \mu \mathrm{s}$. (b) Write enable, VL, and memristors state, $x_{\mathrm{ME} 1}$ and $x_{\mathrm{ME} 2}$, signals.

to $V_{\mathrm{DD}} / 2$. The pulse width is determined by the time required for the memristor to change its state from logic " 1 " $\left(R_{\mathrm{ON}}\right)$ to logic " 0 " $\left(R_{\mathrm{OFF}}\right)$ or vice versa. Waveforms in Fig. 7 illustrate the write operation. In this case $R_{\mathrm{INIT}}=40 \mathrm{k} \Omega$ and the initial state is around 0.6. The diagrams show two write operations, for both when $\mathrm{D}$ is " 1 " and when it is " 0 ." By applying $V_{\mathrm{DD}} / 2$ to $\mathrm{VL}$ line, there will be a $-V_{\mathrm{DD}} / 2$ potential across the memristor ME2 and $V_{\mathrm{DD}}-V_{\mathrm{th}, \mathrm{M} 1}$ across the memristor ME1.

The highlighted area in Fig. 7(b) shows the difference in the write operation between ME1 and ME2. When $D=0$ and $\overline{\mathrm{D}}=$ $V_{\mathrm{DD}}$, there is a threshold voltage $\left(V_{\mathrm{th}}\right)$ drop at the $\overline{\mathrm{SB}}$ node. Thus, the potential across the memristor would be $V_{\mathrm{DD}} / 2-$ $V_{\mathrm{th}, \mathrm{M} 2}$. At the same time, $-V_{\mathrm{DD}} / 2$ is the voltage across the ME1, so the change in state in ME1 occurs faster than memristor ME2. The time for a state change is approximately $75 \mathrm{~ns}$ for ME1 and $220 \mathrm{~ns}$ for ME2. Therefore, $145 \mathrm{~ns}$ delay is imposed because of the voltage drop across the ME2. Fig. 7(b) illustrates simulation results carried out using a behavioral SPICE macromodel.

\section{B. Read Operation}

Let us assume that ME1 and ME2 were programmed as a logic "1" and logic "0," respectively. Therefore, ME1 and ME2 are in the "ON" and "OFF" states and $R_{\mathrm{INIT}, \mathrm{ME} 1}=200 \Omega$ and $R_{\mathrm{INIT}, \mathrm{ME} 2}=99 \mathrm{k} \Omega$. In this case, the search line, $\mathrm{S}$, is activated first. At the same time search select signal, SS, is activated to turn on the two select transistors, M5 and M6. The word select (WS) is disabled during the read operation. Fig. 8 shows the waveforms for a complete read cycle. Read operation requires higher voltage for a short period of time. The VL pulse width (PW) for read operation is $12 \mathrm{~ns}$ as illustrated in Fig. 8(b) which is the "minimum" pulse width necessary to retain memristor's state.

For a matching "1" (when $S=V_{\mathrm{DD}}$ ), the sequence of operations are as follows: 1) match line, ML, is pre-charged; 2) SS is 


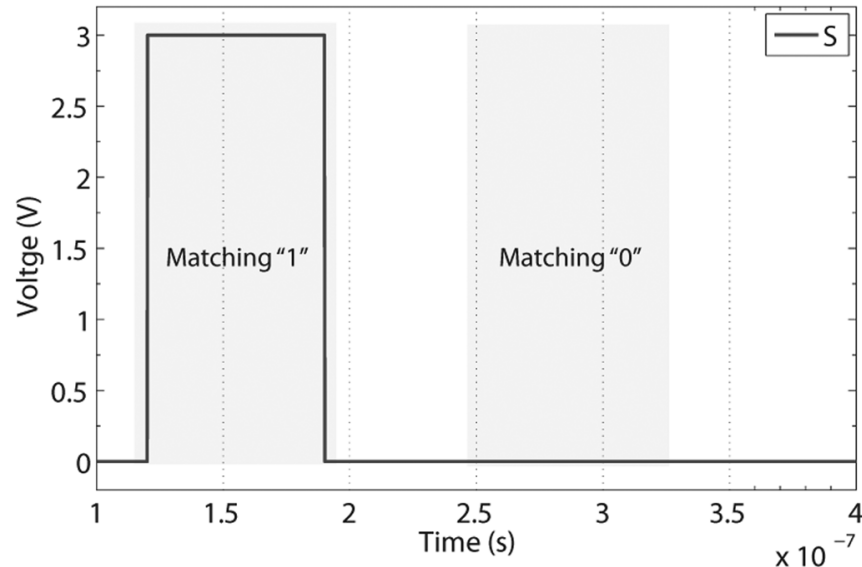

(a)

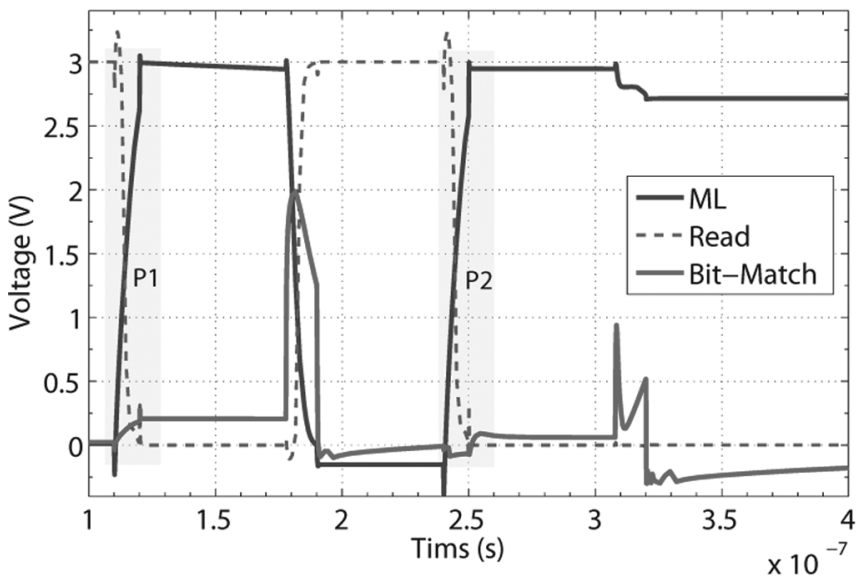

(c)

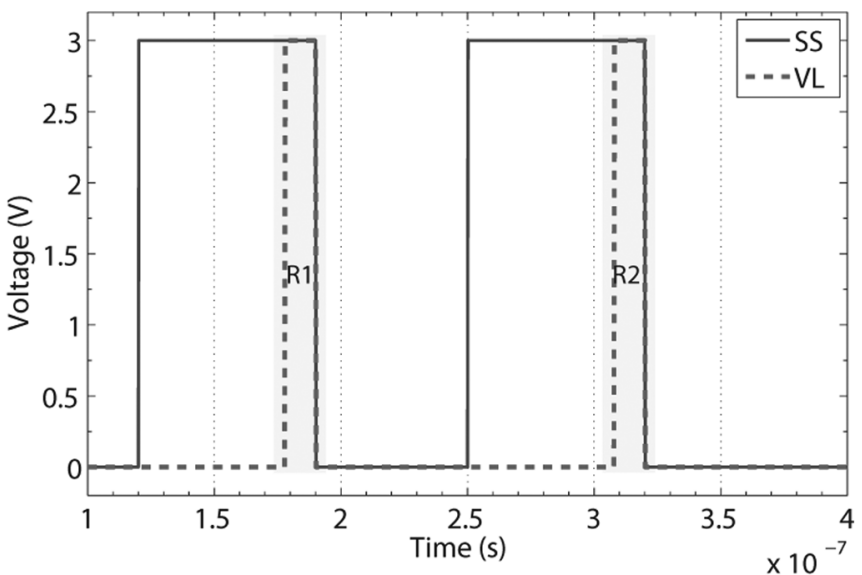

(b)

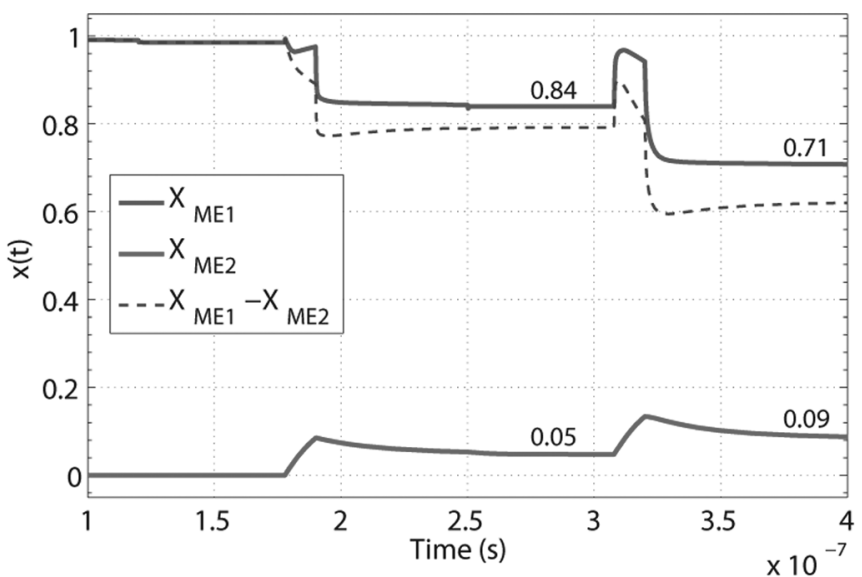

(d)

Fig. 8. Read operation timing diagram: (a) Search signal (S). For matching "1" $S=V_{\mathrm{DD}}$ and for matching "0" $S=0$. (b) Search select (SS) and read enable (VL) signals. $\mathrm{VL}_{\text {active }}=3.0 \mathrm{~V}\left(V_{\mathrm{DD}}\right)$. (c) Bit-match, read, and match-line (ML) signals. Read $=\overline{\mathrm{ML}}$. (d) ME1 and ME2 state variable signals. In (b) and (c), $\mathrm{R} 1, \mathrm{R} 2, \mathrm{P} 1$, and P2 represent two read and match-line pre-charge phases, respectively. The final (stable) values for $x_{\mathrm{ME} 1}$ and $x_{\mathrm{ME} 2}$ after two read operations are around 0.7 and 0.09 . The difference between $x_{\mathrm{ME} 1}$ and $x_{\mathrm{ME} 2}$, in terms of time is also shown in (d).

TABLE III

Comparison Between the Proposed CAM Cells IN Fig. 6

\begin{tabular}{lcccc}
\hline Cell name & $\begin{array}{c}\mathrm{VL}_{\min . P W}, \mathrm{~W} \\
\mathrm{VL}_{\mathrm{W}}=V_{\mathrm{DD}} / 2\end{array}$ & $\begin{array}{c}\mathrm{VL}_{\min } \mathrm{PW}, \mathrm{R} \\
\mathrm{VL}_{\mathrm{R}}=V_{\mathrm{DD}}\end{array}$ & $\begin{array}{c}\mathrm{V}_{\mathrm{drop}} \text { (bit-match) } \\
\text { Voltage [V] }\end{array}$ & $\begin{array}{c}\text { Data \& Search } \\
\text { Buses }\end{array}$ \\
\hline \hline 6-T NOR (Fig. 6(b)) & 223 & 5 & 0 & Separate \\
5-T NOR (Fig. 6(a)) & 219 & 9 & $V_{\text {th }}$ & Separate \\
7-T NOR/NAND (Fig. 6(c/d)) & 220 & 12 & $V_{\text {th }}$ & Shared \\
\hline
\end{tabular}

activated; and 3) VL is enabled as is shown in Fig. 8(a)-(c). A logic " 1 " is transferred to the bit-match node, which discharges the match line, $\mathrm{ML}_{i}$, through transistor ML. At this point $x_{\mathrm{ME1}}$ commences to decrease its state from 1 to 0.84 and $x_{\mathrm{ME} 2}$ increases its state from 0 to 0.05 . Thus, there is a match between stored Data and Search Data. The following read operation for $S=$ "0" follows a similar pattern as shown in Fig. 8(c). The simulation results confirm the functionality of proposed MCAM circuitry.

\section{Simulation Results Analysis}

Table III provides a comparison between the various MCAM cells that are proposed in Fig. 6. It is worth noting that simulations are based on a single cell. Therefore there are no differences in characteristics between 7-T NAND and 7-T NOR cells.
The difference in minimum VL pulse width for read operation $\left(\mathrm{VL}_{\min . P W} \mathrm{R}\right)$, between different MCAM cells, is relatively significant and is brought about as the result of pass-transistors in the path from search line to the bit-match node. One important issue in the design of MCAM cells is endurance. For instance, DRAM cells must be refreshed at least every $16 \mathrm{~ms}$, which corresponds to at least $10^{10}$ write cycles in their life cycle [28]. Analysing a write operation followed by two serial read operations shows that 5-T, 6-T, and 7-T NOR/NAND cells deliver a promising result. After two serial read operations the memristor state values for $x_{\mathrm{ME} 1}$ and $x_{\mathrm{ME} 2}$ are, 0.74 and 0.06 , and 0.71 and 0.09 , for 5-T, 6-T, and 7-T NOR/NAND cell, respectively. The overall conclusion from the simulation results shows that in terms of speed, the 6-T NOR-type MCAM cell has improved performance, but it uses separate Data and Search lines. The 7-T 


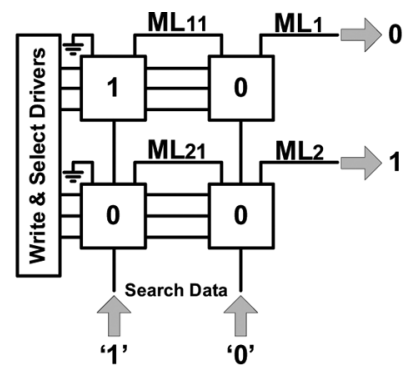

(a)

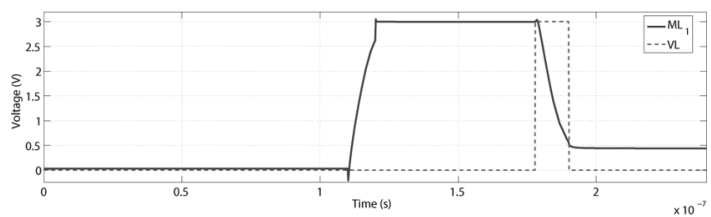

(b)

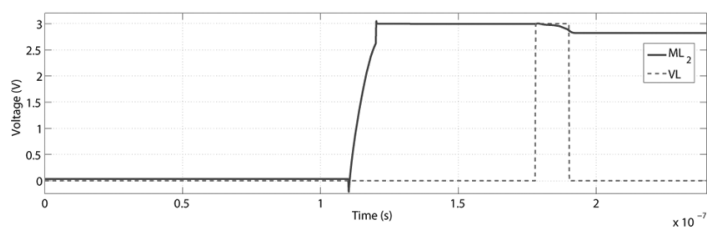

(c)

Fig. 9. $2 \times 2$ MCAM structure. (a) $2 \times 2$ architecture. (b) $\mathrm{ML}_{1}$ signal. (c) $\mathrm{ML}_{2}$ signal. The search data ("10") is matched with the first row stored information so the $\mathrm{ML}_{1}=0$ shows the search data is matched with row 1 and $\mathrm{ML}_{2}=1$ shows the data is not matched with the stored information in the second row ( row $_{2}$ ). (a) $2 \times 2$ architecture, search data ("10"), and matching information. (b) $\mathrm{ML}_{1}$ signal behavior once VL triggers matching operation. (c) $\mathrm{ML}_{2}$ signal behavior once VL triggers matching operation.

NOR/NAND cell shares the same line for Data and Search inputs. However, it is slightly slower $\mathrm{VL}_{\min } . \mathrm{PW}, \mathrm{R}=12 \mathrm{~ns}$, while the swing on the match-line is reduced by threshold voltage $\left(V_{\mathrm{th}}\right)$ drop.

1) Power Analysis: A behavioral model was used to estimate peak, average, and RMS power dissipation of an MCAM cell compared to the conventional SRAM-based cell. The power consumption is the total value for the static and dynamic power dissipation. A reduction of some $96 \%$ in average power consumption with an MCAM cell was noted. The maximum power dissipation reduction is over $74 \%$ for the memristor-based structure. The root mean square (RMS) value of current, which is sunk from the supply rail for the MCAM, is around $47 \mu \mathrm{A}$ less than the conventional SRAM-based circuitry, which shows over $95 \%$ reduction. To the best of our knowledge this is the first power consumption analysis of a memristor-based structure using a behavioral modeling approach. As the technology matures it is conjectured that a similar power source could be used for the hybrid scaled CMOS/Memristor cell.

\section{A $2 \times 2$ Structure Verification}

Fig. 9 illustrates implementation of a $2 \times 2$ structure whereby the 7-T NAND-type [see Fig. 6(d)] is used. As is stated before, in the NOR-type, ML makes a connection between shared ML and ground while in the NAND-type, the ML transistors act as a series of switches between the $\mathrm{ML}_{\text {out }}$ and ground. The $\mathrm{ML}_{1}$ and $\mathrm{ML}_{2}$ match signals, illustrated in Fig. 9(a), are these

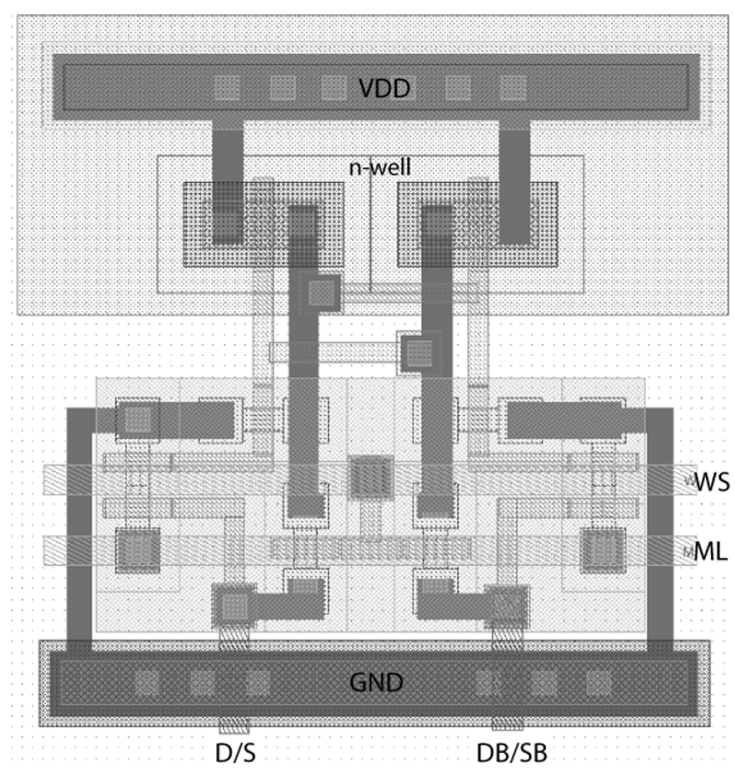

(a)

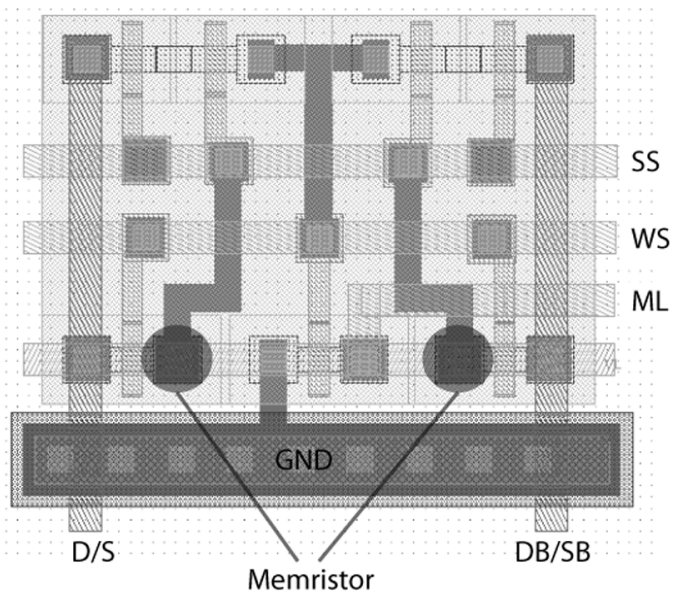

(b)

Fig. 10. Layout implementation (a) conventional SRAM-based and (b) proposed MCAM cells. In (a) $V_{\mathrm{DD}}$ line is required. In (b), highlighted regions show the two memristors in the upper layer. (a) Conventional 10-T NOR-type CAM cell. (b) 7-/T, 2-M NOR-type MCAM cell.

$\mathrm{ML}_{\text {out }}$ signals. The cells are initially programmed to be " 0 " or " 1 " and the search bit vector is "10." The first row cells are programmed "10." As the consequence, $\mathrm{ML}_{1}$ is discharged since there is a match between the stored and search bit vectors. Fig. 9(b) and (c) demonstrate the $\mathrm{ML}_{1}$ and $\mathrm{ML}_{2}$ outputs, respectively. Basically, using the ML transistors as an array of pass-transistors in a NAND-type structure imposes a significant delay, but in this case, the timing information shows the delay of matching process is around $12 \mathrm{~ns}$.

A large scale cosimulation of crossbar memories can be carried out each junction assumed to be either a diode or a 1D-1R (a parallel structure of one diode and one resistor) or even a linear resistor [29]. However, the modeling approach should be carefully revisited since large resistor nonlinearity is associated with crosspoint devices [19]. A cosimulation of crossbar memories, considering the highly nonlinear crosspoint junctions, underpins our longer term research objective. 


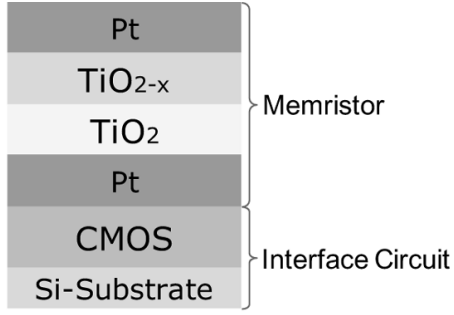

(a)

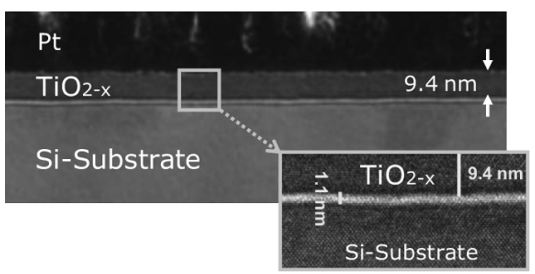

(b)

Fig. 11. Cross sectional view of the memristor-MOS implementation and TEM microphotograph of $\mathrm{TiO}_{2-x}$ deposition. (a) Cross section of memristor-MOS layout. (b) TEM microphotograph.

\section{PHYSICAL LAYOUT AND FABRICATION}

\section{A. Physical Layout}

Layout of conventional 10-T NOR-type CAM and 7-T NORtype MCAM cells are shown in Fig. 10. The MCAM cell has a dimensions of $4.8 \times 4.36 \mu \mathrm{m}^{2}$ while the dimensions for the conventional SRAM-based cell is $6.0 \times 6.5 \mu \mathrm{m}^{2}$. Thus, the reduction in silicon area is in the order of $46 \%$. The $2 \times 2$ structure also shows over a $46 \%$ area reduction. The two memristors, shown in highlighted regions of Fig. 10(b) are implemented between metal-3 and metal-4 layers as part of CMOS post processing.

\section{B. Fabrication and Layer Definitions}

Fig. 11(a) illustrates a cross-section of $\mathrm{Pt}, \mathrm{TiO}_{2}$, and $\mathrm{TiO}_{2-x}$ layers over silicon substrate. The $\mathrm{TiO}_{2}$ layer thickness must be restricted below two nanometers, to prevent separate conduction through the individual layers. The n-type MOS devices are patterned onto a silicon wafer using normal CMOS processing techniques, which subsequently is covered with a protective oxide layer. The Pt memristor wires are patterned and connections made to the n-type MOS devices. The upper Pt nanowire is patterned and, electrical connections made by photolithography (to spatially locate the vias) and aluminum metal deposition [4].

Fig. 11(b) demonstrates a TEM microphotograph of a $\mathrm{TiO}_{2-x}$ overlay on a silicon substrate in order to explore the controllability of oxygen ions. The device consists of a top gate $\mathrm{Pt}, \mathrm{TiO}_{2} / \mathrm{TiO}_{2-x}$ layer and back gate $\mathrm{Pt}$ on $\mathrm{SiO}_{2}$ layer of silicon. $\mathrm{TiO}_{2-x}$ thin film with a thickness of $9.4 \mathrm{~nm}$ was deposited on a silicon wafer using sputtering technique. Table IV is deposition result with sputtering technique. Samples show that $1.85 \%$ oxygen $(\mathrm{O})$ vacancy can be achieved keeping within the $2 \%$ tolerance.
TABLE IV

DEPOSITION RESUlTS USING SPUTTERING TECHNIQUE

\begin{tabular}{c|cccc}
\hline & $\mathrm{O}$ & $\mathrm{Ti}$ & $\mathrm{O}-2 \times \mathrm{Ti}$ & $(\mathrm{O}-2 \times \mathrm{Ti}) / \mathrm{Ti}$ \\
$\%$ & $\%$ & Normalized & Normalized \\
\hline 1 & 66.46 & 33.54 & -0.62 & -1.85 \\
2 & 66.67 & 33.32 & 0.03 & 0.09 \\
\hline
\end{tabular}

\section{CONCLUSION}

The idea of a circuit element, which relates the charge $q$ and the magnetic flux $\phi$ realizable only at the nanoscale with the ability to remember the past history of charge flow, creates interesting approaches in future CAM-based architectures as we approach the domain of multi-technology hyperintegration where optimization of disparate technologies becomes the new challenge. The scaling of CMOS technology is challenging below $10 \mathrm{~nm}$ and thus nanoscale features of the memristor can be significantly exploited. The memristor is thus a strong candidate for tera-bit memory/compare logic.

The non-volatile characteristic and nanoscale geometry of the memristor together with its compatibility with CMOS process technology increases the memory cell packing density, reduces power dissipation and provides for new approaches towards power reduction and management through disabling blocks of MCAM cells without loss of stored data. Our simulation results show that the MCAM approach provides a $45 \%$ reduction in silicon area when compared with the SRAM equivalent cell. The Read operation of the MCAM ranges between 5 to $12 \mathrm{~ns}$, for various implementations, and is comparable with current SRAM and DRAM approaches. However the Write operation is significantly longer.

Simulation results indicate a reduction of some $96 \%$ in average power dissipation with the MCAM cell. The maximum power reduction is over $74 \%$ for the memristor-based structure. The RMS value of current sunk from the supply rail for the MCAM is also approximately $47 \mu \mathrm{A}$, which correspond to over a $95 \%$ reduction when compared to SRAM-based circuitry. To the best of our knowledge this is the first power consumption analysis of a memristor-based structure that has been presented using a behavioral modeling approach. As the technology is better understood and matures further improvements in performance can be expected

\section{ACKNOWLEDGMENT}

The authors would like to thank iDataMap Pty Ltd for the initial concept and gratefully acknowledge Dr. J. W. Kim, Dr. H. H. Kim, and Dr. B. J. Lee of NanoFab in Korea Advanced Institute Science and Technology (KAIST) for their contribution towards fabrication.

\section{REFERENCES}

[1] G. I. Bourianoff, P. A. Gargini, and D. E. Nikonov, "Research directions in beyond CMOS computing," Solid-State Electron., vol. 51, no. 11-12, pp. 1426-1431, 2007.

[2] D. Akinwande, S. Yasuda, B. Paul, S. Fujita, G. Close, and H. S. P. Wong, "Monolithic integration of CMOS VLSI and CNT for hybrid nanotechnology applications," in Proc. 38th Eur. Solid-State Device Res. Conf. (ESSDERC), 2008, pp. 91-94.

[3] N. Engheta, "Circuits with light at nanoscales: Optical nanocircuits inspired by metamaterials," Science, vol. 317, no. 5845, pp. 1698-1702, 2007. 
[4] D. B. Strukov, G. S. Snider, D. R. Stewart, and R. S. Williams, "The missing memristor found," Nature, vol. 453, no. 7191, pp. 80-83, 2008.

[5] L. O. Chua, "Memristor-The missing circuit element," IEEE Trans. Circuits Syst., vol. 18, no. 5, pp. 507-519, Sep. 1971.

[6] S. M. Kang, "On the modeling of some classes of nonlinear devices and systems," Ph.D. dissertation, Dept. Elect. Electron. Eng., Univ. California, Berkeley, CA, 1975.

[7] L. O. Chua and S. M. Kang, "Memristive devices and systems," Proc. IEEE, vol. 64, no. 2, pp. 209-223, Feb. 1976.

[8] O. Kavehei, Y. S. Kim, A. Iqbal, K. Eshraghian, S. F. Al-Sarawi, and D. Abbott, "The fourth element: Insights into the memristor," in Proc. IEEE Int. Conf. Commun., Circuits Syst. (ICCCAS), Jul. 2009, pp. 921-927.

[9] J. J. Yang, M. D. Pickett, X. Li, D. A. A. Ohlberg, D. R. Stewart, and R. S. Williams, "Memristive switching mechanism for metal-oxide-metal nanodevices," Nature Nanotechnol., vol. 3, no. 7, pp. 429-433, 2008.

[10] D. B. Strukov and R. S. Williams, "Exponential ionic drift: Fast switching and low volatility of thin-film memristors," Appl. Phys. A: Mater. Sci. Process., vol. 94, no. 3, pp. 515-519, 2009.

[11] Z. Biolek, D. Biolek, and V. Biolková, "SPICE model of memristor with nonlinear dopant drift," Radioeng. J., vol. 18, no. 2, pp. 211-211, 2009.

[12] S. Benderli and T. A. Wey, "On SPICE macromodelling of $\mathrm{TiO}_{2}$ memristors," Electron. Lett., vol. 45, no. 7, pp. 377-379, 2009.

[13] Y. N. Joglekar and S. J. Wolf, "The elusive memristor: Properties of basic electrical circuits," Eur. J. Phys., vol. 30, no. 4, pp. 661-661, 2009.

[14] S. Shin, K. Kim, and S. M. Kang, "Compact models for memristors based on charge-flux constitutive relationships," IEEE Trans. Comput.Aided Des. Integr. Circuits Syst., vol. 29, no. 4, pp. 590-598, Apr. 2010.

[15] Emerging Research Devices (ERD), "International Technology Roadmap for Semiconductors" 2009, Ed. [Online]. Available: http://www.itrs.net/

[16] R. F. Freitas and W. W. Wilcke, "Storage-class memory: The next storage system technology," IBM J. Res. Develop., vol. 52, no. 4-5, pp. 439-448, 2008.

[17] P. J. Kuekes, D. R. Stewart, and R. S. Williams, "The crossbar latch: Logic value storage, restoration, and inversion in crossbar circuits," $J$. Appl. Phys., vol. 97, pp. 034301-034301, 2005.

[18] D. B. Strukov and R. S. Williams, "Four-dimensional address topology for circuits with stacked multilayer crossbar arrays," Proc. Nat. Academy Sci., vol. 106, no. 48, pp. 20155-20158, 2009.

[19] P. O. Vontobel, W. Robinett, P. J. Kuekes, D. R. Stewart, J. Straznicky, and R. S. Williams, "Writing to and reading from a nano-scale crossbar memory based on memristors," Nanotechnol., vol. 20, no. 42, pp. 425204-425204, 2009.

[20] O. Tyshchenko and A. Sheikholeslami, "Match sensing using matchline stability in content-addressable memories (CAM)," IEEE J. SolidState Circuits, vol. 43, no. 9, pp. 1972-1981, Sep. 2008.

[21] T. Kumaki, Y. Kuroda, M. Ishizaki, T. Koide, H. J. Mattausch, H. Noda, K. Dosaka, K. Arimoto, and K. Saito, "Real-time Huffman encoder with pipelined CAM-based data path and code-word-table optimizer," IEICE-Trans. Inf. Syst., vol. E90-D, no. 1, pp. 334-345, 2007.

[22] Y. D. Kim, H. S. Ahn, S. Kim, and D. K. Jeong, "A high-speed range-matching TCAM for storage-efficient packet classification," IEEE Trans. Circuits Syst. I, Reg. Papers, vol. 56, no. 6, pp. 1221-1230, Jun. 2009.

[23] N. Verma and A. P. Chandrakasan, "A $256 \mathrm{~kb} \times 65 \mathrm{~nm} 8 \mathrm{~T}$ subthreshold SRAM employing sense-amplifier redundancy," IEEE J. Solid-State Circuits, vol. 43, no. 1, pp. 141-149, Jan. 2008.

[24] S. K. Lu and C. H. Hsu, "Fault tolerance techniques for high capacity RAM," IEEE Trans. Reliab., vol. 55, no. 6, pp. 293-306, Jun. 2006.

[25] N. Mohan and M. Sachdev, "Low-leakage storage cells for ternary content addressable memories," IEEE Trans. Very Large Scale Integr. (VLSI) Syst., vol. 17, no. 5, pp. 604-612, May 2009.

[26] K. Pagiamtzis and A. Sheikholeslami, "Content-addressable memory (CAM) circuits and architectures: A tutorial and survey," IEEE J. SolidState Circuits, vol. 41, no. 3, pp. 712-727, Mar. 2006.

[27] K. Witrisal, "Memristor-based stored-reference receiver-The UWB solution?," Electron. Lett., vol. 45, no. 14, pp. 713-714, 2009.

[28] D. L. Lewis and H. H. S. Lee, "Architectural evaluation of 3D stacked RRAM caches," in Proc. IEEE Int. Conf. 3D Syst. Integr. (3DIC), San Francisco, CA, 2009, pp. 1-4.

[29] M. M. Ziegler and M. R. Stan, "CMOS/nano co-design for crossbarbased molecular electronic systems," IEEE Trans. Nanotechnol., vol. 2, no. 4, pp. 217-230, Dec. 2003.

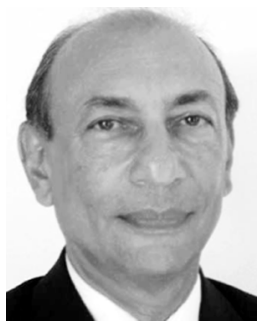

Kamran Eshraghian received the Ph.D., M.Eng.Sc., and B.Tech. degrees from the University of Adelaide, South Australia, and the Dr.-Ing e.h., (Doctor of Engineering) in integration of nanoelectronics with that of light wave technology from the University of Ulm, Germany, in 2004.

$\mathrm{He}$ is best known in international arena as being one of the fathers of CMOS VLSI having influenced two generations of researchers in both academia and industry. In 1979, he joined the Department of Electrical and Electronic Engineering, University of Adelaide, after spending some ten years with Philips Research, both in Australia and Europe. In 1994, he was invited to take up the Foundation Chair of Computer, Electronics and Communications Engineering in Western Australia, and became Head of School of Engineering and Mathematics and Distinguished University Professor and subsequently became the Director of Electron Science Research Institute. In 2004, he became founder/President of Elabs as part of his vision for horizontal integration of nanoelectronics with those of bio and photon-based technologies, thus creating a new design domain for system on system $(\mathrm{SoS})$ integration. Currently, he is the President of Innovation Labs and also serves as the Chairman of the Board of Directors of four high technology companies. In 2007, he was visiting Professor of Engineering and the holder of inaugural Ferrero Family Chair in Electrical Engineering at UC Merced prior to his move in 2009 to Chungbuk National University, Korea, as Distinguished Professor, World Class University (WCU) program. He has held a number of professoriate appointments in US and in Europe, has coauthored six textbooks and has lectured widely in VLSI and multitechnology systems. He has founded six high technology companies, providing intimate links between university research and industry.

Prof. Eshraghian is a Fellow of the Institution of Engineers, Australia.

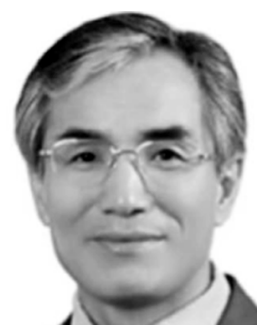

Kyoung Rok Cho (S'89-M'92) received the B.S. degree in electronic engineering from Kyoungpook National University, Taegu, Korea, in 1977, and the M.S. and $\mathrm{Ph} . \mathrm{D}$. degrees in electrical engineering from University of Tokyo, Tokyo, Japan, in 1989 and 1992, respectively.

From 1979 to 1986, he was with the TV Research Center, LG Electronics, Korea. He is currently a Professor with the College of Electrical and Computer Engineering, Chungbuk National University, Korea. His research interests include the field of high-speed and low-power circuit design, SoC platform design for communication systems, and prospective CMOS image sensors. He is also currently director of the World Class University (WCU) Program, Chungbuk National University, Korea. His research interests include memristor and memristive systems, and design of multi-layer system-on-systems ( $\mathrm{SoS})$ technology.

Prof. Cho was a recipient of IEEK Award in December 2004. During 1999 and 2006, he spent 2 years at Oregon State University, as a Visiting Scholar. He is a member of the Institute of Electronics Engineers of Korea (IEEK).

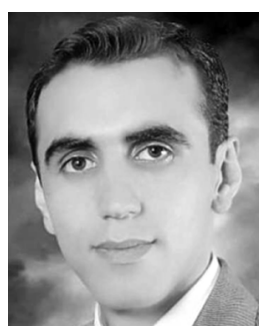

Omid Kavehei (S'05) received the M.S. degree in computer systems engineering from the National University of Iran (Shahid Beheshti University), Tehran, Iran, in 2005 . He is currently pursuing the $\mathrm{Ph} . \mathrm{D}$. degree in electrical and electronic engineering from the University of Adelaide, South Australia, under Dr. S. Al-Sarawi and Prof. D. Abbott.

He was a Visiting Scholar with the Chungbuk National University (CBNU), South Korea, under Prof. K. Eshraghian and Prof. K. R. Cho in 2009-2010. His research interests include variation-aware low-power mixed-signal circuits and systems design methodologies; emerging non-volatile memory systems, especially memristor-based crossbar architecture; hardwareoriented cognitive matching structures, particularly human-like intelligent VLSI pattern recognition systems; bio-inspired information processing and unconventional computing; applications of memristive devices in artificial intelligent; and VLSI design aspects of computer arithmetic modules.

Mr. Kavehei was a recipient of an Endeavour International Postgraduate Research (Ph.D.) Scholarship (EIPRS), DEEWR, Australian Government, in 2008 and the D. R. Stranks Travelling Fellowship and the World Class University (WCU) Program Scholarship to fabricate two types of high-speed and high-dynamic range CMOS Image Sensors in collaboration with Samsung Semiconductor through the Chungbuk National University. He is a student member of the Engineers Australia (EA) and a committee member of the Electrical and Electronic Engineering Society of Adelaide University (EEESAU). 


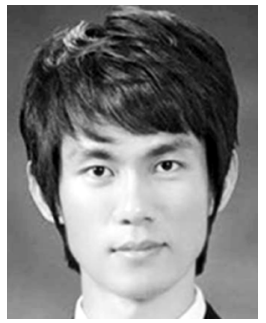

Soon Ku Kang received the B.S. degree in electronics engineering from Chungbuk National University, Cheongju, South Korea, in 2009, where he is currently pursuing the M.S. degree in the Department of Information and Communication.

His research interest is in the area of memory devices and low-voltage low-power CMOS analog circuits.

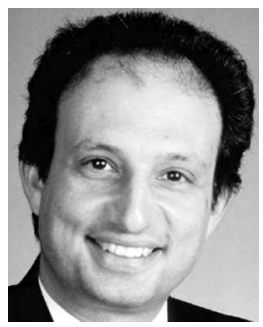

Derek Abbott (M'85-SM'99-F'05) was born in South Kensington, London, U.K., on May 3, 1960. $\mathrm{He}$ received the B.S. degree (honors) in physics from the Loughborough University of Technology, U.K., in 1982 and the Ph.D. degree (with commendation) in electrical and electronic engineering from the University of Adelaide, Adelaide, Australia, in 1995, under K. Eshraghian and B. R. Davis.

He has led a number of research programs in the imaging arena, ranging from the optical to infrared to millimeter wave to T-ray (terahertz gap) regimes. From 1978 to 1986, he worked at the GEC Hirst Research Centre, London, U.K., in the area of visible and infrared image sensors. His expertise also spans VLSI design, optoelectronics, device physics, and noise, where he has worked with nMOS, CMOS, SOS, CCD, GaAs, and vacuum microelectronic technologies. On migration to Australia, he worked for Austek Microsystems, Technology Park, South Australia, in 1986. Since 1987, he has been with the University of Adelaide, where he is presently a Full Professor with the School of Electrical and Electronic Engineering. He has appeared on national and international television and radio and has also received scientific reportage in New Scientist, The Sciences, Scientific American, Nature, The New York Times, and Sciences et Avenir. He holds over 350 publications/patents and has been an invited speaker at over 80 institutions, including Princeton University, NJ, MIT, MA, Santa Fe Institute, NM, Los Alamos National Laboratories, NM, Cambridge University, U.K., and EPFL, Lausanne, Switzerland.

Prof. Abbott is a Fellow of the Institute of Physics (IOP), with honorary life membership. He is a recipient of the GEC Bursary (1977), the Stephen Cole Elder Prize (1998), the E.R.H. Tiekink Memorial Award (2002), the SPIE Scholarship Award for Optical Engineering and Science (2003), the South Australian Tall Poppy Award for Science (2004), and the Premier's SA Great Award in Science and Technology for outstanding contributions to South Australia (2004). He has served as an Editor and/or a Guest Editor for a number of journals including IEEE Journal OF SOLID-STATE CIRCUITS, Chaos (AIP), Smart Structures and Materials (IOP), the Journal of Optics B (IOP), the Microelectronics Journal (Elsevier), Fluctuation Noise Letters (World Scientific), and is currently on the Editorial Board of the PROCEEDING OF THE IEEE. He has served on a number of IEEE technical program committees, including the IEEE APCCS and the IEEE GaAs IC Symposium. Prof. Abbott coedited Quantum Aspects of Life, Imperial College Press (ICP) and coauthored Stochastic Resonance, Cambridge University Press (CUP).

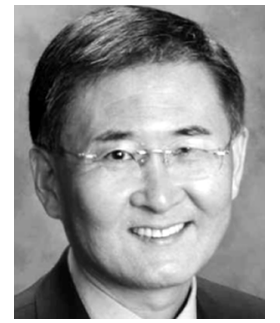

Sung-Mo Steve Kang (S'73-M'75-SM'80-F'90) received the B.S. degree from Fairleigh Dickinson University, Teaneck, NJ, in 1970, the M.S. degree from the State University of New York at Buffalo, Buffalo, NY, in 1972, and the Ph.D. degree from the University of California, Berkeley, in 1975, all in electrical engineering.

From 1995 to 2000, he was the Head of the Department of Electrical and Computer Engineering, University of Illinois at Urbana-Champaign (UIUC). Prior to UIUC, he was a Supervisor of High-End Microprocessor Design with AT\&T Bell Laboratories, Murray Hill, NJ, and also has served as a Faculty Member of Rutgers University, New Brunswick, NJ. He was a Visiting Professor at the University of Karlsruhe in 1997, the Technical University of Munich in 1998, KAIST in 2003, and the Swiss Federal Institute of Technology, Lausanne, in 1989 and 2006. From 2001 to 2007, he was the Dean of Baskin School of Engineering and Professor of Electrical Engineering at UC Santa Cruz. He is currently the Chancellor and Professor of Engineering with the School of Engineering, UC Merced. He holds 15 patents, published over 350 papers, and coauthored nine books His research interest includes low-power very large scale integration design; mixed-signal mixed-technology integrated system; modeling and simulation of semiconductor devices and circuits; high-speed optoelectronic circuits and systems; and bioelectronic circuits and systems.

Prof. Kang has served on the Editorial Boards of the PROCEEDING OF THE IEEE, the IEEE TRANSACTIONS ON CIRCUITS AND SYSTEMS, the International Journal of Circuit Theory and Applications, and Circuits, Signals and Systems, as the Founding Editor-in-Chief of the IEEE TRANSACTIONS ON VERY LARGE SCALE (VLSI) SYSTEMS and as the President of the IEEE Circuits and Systems (CAS) Society in 1991. He was inducted into the Silicon Valley Engineering Council Hall of Fame in 2009. He was a recipient of the IEEE Circuits and Systems Society Meritorious Service Award in 1994, the Humboldt Research Award for Senior U.S. Scientists in 1996, the IEEE Graduate Teaching Technical Field Award in 1996, the IEEE CAS Society Technical Achievement Award in 1997, the Korean Broadcasting System Award in Industrial Technology in 1998, the Semiconductor Research Corporation Technical Excellence Award in 1999, the IEEE CAS Society Golden Jubilee Medal in 1999, the IEEE Third Millennium Medal in 2000, the Outstanding Alumnus Award in Electrical Engineering, UC Berkeley, in 2001, the Mac E. Van Valkenburg Society Award of IEEE Circuits and Systems Society in 2005, the Chang-Lin Tien Education Leadership Award in 2007, the International Society for Quality Electronic Design Quality Award in 2008, and the Korean-American Leadership Award in 2008. He is a Fellow of the Association for Computing Machinery and the American Association for the Advancement of Science. 